Canadian

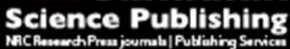

Applied Physiology, Nutrition, and Metabolism Physiologie appliquée, nutrition et métabolisme

\title{
Effect of Acute Nitrate Supplementation on Neurovascular Coupling and Cognitive Performance in Hypoxia
}

\begin{tabular}{|r|l|}
\hline Journal: & Applied Physiology, Nutrition, and Metabolism \\
\hline Manuscript ID & apnm-2015-0400.R1 \\
\hline Manuscript Type: & Article \\
\hline Complete List of Authors: & $\begin{array}{l}\text { Lefferts, Wesley; Syracuse University, Exercise Science } \\
\text { Hughes, William; Syracuse University, Exercise Science; University of } \\
\text { Iowa, Physical Therapy and Rehabilitation Science } \\
\text { White, Corey; Syracuse University, Psychology } \\
\text { Brutsaert, Tom; Syracuse University, Exercise Science } \\
\text { Heffernan, Kevin; Syracuse University, Exercise Science }\end{array}$ \\
\hline Keyword: & nitrate, neurovascular coupling, cognition, hypoxia \\
\hline & \\
\hline
\end{tabular}


7 William E Hughes, william-e-hughes@uiowa.edu

8 Corey N White, cnwhite@syr.edu

9 Tom D Brutsaert, tdbrutsa@syr.edu

10 Kevin S Heffernan, ksheffer@syr.edu

Running head: Nitrate, neurovascular coupling, and cognition in hypoxia

\section{* Corresponding Author:}

Wesley K Lefferts, MS

The Human Performance Laboratory

Department of Exercise Science

Syracuse University

Syracuse NY, 13244, USA

P: 503-804-4424

wleffert@syr.edu

\footnotetext{
${ }^{1}$ William Hughes currently works at University of lowa, lowa City, IA.
} 


\section{Abstract}

The matching of oxygen supply to neural demand (i.e. neurovascular coupling [NVC]) is an important determinant of cognitive performance. The impact of hypoxia on NVC remains poorly characterized. NVC is partially modulated by nitric oxide (NO) which may initially decrease in hypoxia. This study

37 investigated the effect of acute NO-donor (nitrate) supplementation on NVC and cognitive function in hypoxia. Twenty healthy men participated in this randomized, double-blind, crossover design study. Following normoxic cognitive/NVC testing, participants consumed either nitrate (NIT) or a NIT-depleted placebo (PLA). Participants then underwent $120 \mathrm{~min}$ of hypoxia $\left(11.6 \pm 0.1 \% \mathrm{O}_{2}\right)$ and all cognitive/NVC testing was repeated. NVC was assessed as change in middle cerebral artery (MCA) blood flow during a cognitive task (incongruent Stroop) using Transcranial Doppler. Additional computerized cognitive testing assessed separately targeted memory, executive function, attention, sensorimotor and social cognition domains. Salivary nitrite significantly increased following supplementation in hypoxia for NIT $(+2.6 \pm 1.0 \mathrm{AU})$ compared to PLA $(+0.2 \pm 0.3 \mathrm{AU} ; \mathrm{p}<0.05)$. Memory performance $(-6 \pm 13$ correct) significantly decreased $(p<0.05)$ in hypoxia while all other cognitive domains were unchanged in hypoxia

47 for both PLA and NIT conditions ( $p>0.05$ ). MCA flow increased during Stroop similarly in normoxia (PLA $\left.+5 \pm 6, \mathrm{NIT}+7 \pm 7 \mathrm{~cm} \cdot \mathrm{s}^{-1}\right)$ and hypoxia $\left(\mathrm{PLA}+5 \pm 9, \mathrm{NIT}+6 \pm 7 \mathrm{~cm} \cdot \mathrm{s}^{-1}\right)(\mathrm{p}<0.05)$ and this increase was not altered by PLA or NIT ( $p>0.05)$. In conclusion, acute hypoxia resulted in significant reductions in memory concomitant with preservation of executive function, attention, and sensorimotor function. Hypoxia

51 had no effect on NVC. Acute NIT supplementation had no effect on NVC or cognitive performance in 52 hypoxia. 


\section{Introduction}

Normal cerebral function is a critical determinant of cognitive performance and is dependent on oxygen supply delivered by the cerebrovasculature (Chen et al. 2013). As brain activity increases, blood flow must increase to meet neural/metabolic demands and support cognitive activity (Vingerhoets and Stroobant 1999), a process known as neurovascular coupling (NVC) (ladecola 2004; Attwell et al. 2010). In hypoxic environments, reductions in arterial oxygen saturation are offset by compensatory vasodilation (Casey and Joyner 2011) and subsequent augmented blood flow (Jia et al. 2011; Willie et al. 2014), the degree of which is proportional to the degree of hypoxemia. In the cerebrovasculature, this compensatory hyperemia manifests as increased extrancranial (i.e. carotid) (Lewis et al. 2014a; Lewis et al. 2014b) and intracranial (i.e. cerebral) blood flow compared to normoxia (Fan et al. 2010). Blood flow regulation in this setting may be partially controlled by nitric oxide (NO) (Van Mil et al. 2002). NO is released from the vascular endothelium and acts to relax vascular smooth muscle, eliciting vasodilation and increased blood flow (Nichols and O'Rourke 2005). In addition to its central role as a regulator of blood flow (Levett et al. 2011), NO ensures optimal hyperemic response to neural activity (NVC) (Attwell et al. 2010), is necessary for hypoxia-induced cerebral vasodilation (Van Mil et al. 2002), and appears to play an integral role in adaptations to hypoxia (Levett et al. 2011). Upon initial exposure to systemic hypoxia (e.g. acute ascent to high altitude), endothelial dysfunction and reductions in NO production have been noted (Duplain et al. 2000). Consequently, reductions in NO bioavailability with hypoxic exposure may interfere with NVC and thereby impact cognitive performance.

Dietary nitrate supplementation may be a novel means of favorably impacting NVC and cognitive function with acute systemic hypoxia exposure. NO can be synthesized endogenously by NO synthases or from the nitrate-nitrite-NO pathway; dietary nitrate, a natural ingredient of beetroots, vegetables, and leafy greens can be reduced to nitrate and increase NO formation (Hord et al. 2009). Unlike the enzymatic reaction to produce NO, the conversion of nitrite to NO takes place preferentially 
81 under hypoxic conditions (Lundberg et al. 2008). The brain can use nitrate as a direct functional source

82 of NO (Piknova et al. 2011). Increased nitrite concentration via dietary nitrate consumption enhances

83 blood flow at rest (Presley et al. 2011) and increases endothelial function and NO production during

84 neuronal activity, augmenting subsequent NVC during increased cognitive demand (Aamand et al. 2013)

85 which may explain select improvements in cognitive function following acute nitrate supplementation in

86 normoxia (Wightman et al. 2015). Thus, dietary nitrate supplementation in hypoxia might improve NVC

87 and thereby attenuate decrements in hypoxia-sensitive cognitive domains, but this has yet to be

88 explored.

The purpose of this study was to investigate the effects of acute nitrate supplementation on

NVC (operationally defined as change in cerebral blood flow from rest to cognitive engagement) and

91 global cognitive function in hypoxia. It was hypothesized that compared to an inert placebo, nitrate

92 supplementation would enhance NVC (manifesting as greater increases in cerebral blood flow measured during cognitive engagement) and improve cognitive function in hypoxia.

\section{Materials and Methods}

\section{Participants}

Twenty-four recreationally active men in overall good health were recruited from the local University community for this study. Two participants were lost to follow-up and one participant experienced a syncopal episode upon hypoxic exposure and could not complete the study. Additionally, one participant was excluded from data analysis due to excessive time between trials ( $>4$ weeks), leaving 20 participants $\left(23 \pm 3 \mathrm{yrs}\right.$, BMI $24.6 \pm 2.8 \mathrm{~kg} \cdot \mathrm{m}^{-2}$, body fat $13.3 \pm 6.8 \%$, hemoglobin $14.7 \pm 1.5$ $\mathrm{g} / \mathrm{dL}$ ) for final analyses. Exclusion criteria included self-reported (determined from a health history questionnaire) smoking, hypertension, diabetes mellitus, hyperlipidemia, pulmonary disease, renal disease, neurological disease, or peripheral artery disease. Hemoglobin concentration was assessed at baseline via finger-stick blood sample and microcuvette (The Hemocue Hemoglobin System, Hb201+; 
105

106

107

108

109

110

111

112

113

Angelholm, Sweden) to screen for anemia (defined as $\mathrm{Hb}<13.5 \mathrm{~g} / \mathrm{dL}$ ). Participants were not taking any medications at the time of the study and were asked to refrain from dietary supplement use for the duration of the study. This study was approved by the Syracuse University Institutional Review Board and all participants provided written informed consent prior to study initiation. Testing was conducted at the same time of day within-participants in a temperature-controlled laboratory. Participants were instructed to fast for $\geq 3$ hours and avoid vigorous exercise and avoid consuming caffeine and alcohol the day of testing. Additionally, participants were given a list of high-nitrate foods to avoid for the 2 days prior to experimental testing.

\section{Design}

This study utilized a randomized, double-blind, crossover-design. All participants completed two experimental trials separated by at least $72 \mathrm{hrs}$. Trials consisted of ingesting either $0.45 \mathrm{~g}$ nitrate bolus (Beet It Sports Shot; NIT) or an inert placebo (PLA) prior to hypoxic exposure. On the second experimental visit participants underwent the opposite treatment condition (i.e. if they received NIT on the first visit then they received PLA on the subsequent visit and vice versa). Both supplements were identical in appearance, taste, volume $(70 \mathrm{~mL})$ and caloric value $(4 \mathrm{~g}$ protein, $20 \mathrm{~g}$ carbohydrate, $0.2 \mathrm{~g}$ fat, $<0.5 \mathrm{~g}$ fiber, $<0.1 \mathrm{~g}$ sodium; Beet It, James White Ltd, Ipswich), however PLA was depleted of nitrate through a specialized manufacturing process (previously reported nitrate content: placebo $\approx 0.01 \mathrm{mmol}$ vs nitrate-rich $\approx 5.0 \mathrm{mmol}$ (Muggeridge et al. 2013b)). The dose of nitrate used in this study has been previously reported to significantly increase plasma nitrate and nitrite concentration and elicit biological effects in normoxia (Muggeridge et al. 2013a; Wightman et al. 2015), and hypoxia (2,500 m)(Muggeridge et al. 2013b) and is in-line with manufacturer recommendations.

Height and weight was assessed via stadiometer and electronic scale, respectively, and body composition was estimated via air displacement plethysmography (BodPod; COSMED, Concord, CA). 
128 Upon arrival to the laboratory, participants rested in the supine position for 10 minutes before

129 normoxic-baseline vascular and cognitive measures were assessed. Vascular measures were collected in 130 the supine position and in the same order under normoxic and hypoxic conditions. After completion of

131 vascular measures participants underwent salivary nitrite testing as a marker of nitrate metabolism,

132 followed by cognitive testing. Participants then ingested either a) NIT or b) PLA immediately prior to 133 entering the normobaric hypoxic chamber $\left(\mathrm{FiO}_{2} 11.6 \pm 0.1 \%, \approx 4,600 \mathrm{~m}\right.$; Hypoxico Systems, New York, 134 NY). Oxygen concentration was measured using an oxygen monitor (PureAire Monitoring Systems Inc., 135 Lake Zurich, IL) secured inside the hypoxic chamber. Our hypoxic stimulus was chosen based on 136 previous research that established $4000-5000 \mathrm{~m}$ as the critical altitude for changes in cognitive function 137 (Babbar and Agarwal 2012). Participants remained in hypoxia for 105 minutes before undergoing 138 hypoxic-vascular and cognitive testing. Our timeline was designed such that cognitive testing would 139 occur concordant with peak nitrate availability ( $\approx 2$ hrs post-nitrate ingestion) as suggested by previous 140 literature (Presley et al. 2011).

141 Measures

142 Quantifying the Hypoxic Stimulus

143 Arterial oxygen saturation was assessed using a reflectance pulse oximeter placed on the 144 forehead (Nonin Medical, Plymouth, $\mathrm{MN}$ ) in order to quantify the hypoxic stimulus. End-tidal $\mathrm{CO}_{2}$

$145\left(\mathrm{EtCO}_{2}\right)$ was measured to ensure $\mathrm{CO}_{2}$ levels were comparable between treatments (Nellcor OxiMax, 146 Covidien, Mansfield, MA) at normoxic and hypoxic baselines with sampling lines secured directly under 147 the nostrils. Participants rested for 10 minutes prior assessment to ensure stable resting values. Data 148 was collected over a 5-minute period with triplicate measures taken during minutes 2-4 and averaged.

149 Salivary Nitrite 
Salivary nitrite was qualitatively assessed using salivary test strips (Berkeley Test, Berkeley, CA).

151 A salivary absorbent pad was placed under the tongue for 3-5 $\mathrm{s}$ and then pressed against a reagent strip.

152 The resulting color was compared to a colored scale to qualitatively assess nitrite availability. Nitrite

153 availability was assessed in 1) normoxia to ensure all participants began with similar, low levels of

154 salivary nitrite; and 2 ) hypoxia ( $\approx 2$ hours after nitrate ingestion) to document changes in nitrite

155 availability following supplementation.

156

\section{Neurovascular Coupling}

Change in cerebral blood flow measured during cognitive perturbations has been reported to reflect changes in cerebral metabolism (i.e. NVC), and the middle cerebral artery (MCA) is the most commonly interrogated vessel in functional transcranial Doppler studies (Sorond et al. 2013; Bakker et al. 2014; Lupo et al. 2015; Payne et al. 2015; Wolf 2015). This vessel was chosen since the MCA accounts for roughly $80 \%$ of total brain blood flow to numerous regions of the brain, including regions essential for executive functions (assessed during our NVC protocol described below). We further assessed common carotid artery (CCA) flow during cognitive perturbation to compliment MCA measures since some regulation of intracranial flow may occur extracranially (Willie et al. 2014). Previous data from our laboratory (unpublished observation) has revealed that there are no significant differences between primary outcome measures across three time points during cognitive perturbation (Stroop task). Thus, CCA blood flow (described below) was assessed once during the Stroop task beginning 30-seconds after task initiation. All NVC metrics were calculated as absolute change from baseline.

In this study we measured NVC during a computerized, modified incongruent Stroop color-word interference task (E-Prime, Psychology Software Tools Inc, Sharpsburg, PA). This test has been previously used as a mental stressor and means of assessing NVC in our laboratory (Heffernan et al. 2014). All participants were familiarized with the Stroop task prior to experimental testing in order to 
173 control for learning effects. The Stroop task was completed in the supine position with the head tilted

174 slightly back, thereby optimizing the imaging window of the carotid artery. The viewing display for

175 normoxic testing was a specialized wall-mounted 107-cm flatscreen television that extended over the

176 participant. Font was displayed approximately $102-\mathrm{cm}$ above the participant with $3.0-\mathrm{cm}$ font on a black

177 background. For hypoxic testing, the Stroop task was projected onto the ceiling of the chamber using a

178 computer-interfaced projector (IN1100, InFocus, Portland, OR) that displayed the task approximately

179 160-cm above the participant with 4.5-cm font. Despite the task being displayed farther away from the

180 participant in hypoxia, the ratio between viewing distance $(\mathrm{cm})$ and font size $(\mathrm{cm})$ was comparable to

181 normoxia (34:1 normoxia, 36:1 hypoxia).

The NVC protocol began when participants were presented with a white crosshair in the center

of the viewing window for approximately 3-seconds. A target word was displayed in incongruous colors

184 (e.g. the word "blue" written in the color red), with four names of response colors presented similarly

185 (e.g. the word "red" written in the color blue). The task was to use a response clicker to identify the

186 color that matched the target word displayed as quickly as possible. The response colors (1-4)

187 corresponded to the remote clicker buttons (1-4) which the participant manipulated using the digits on

188 their dominant side (index finger - pinky finger). This task lasted 4-minutes in duration, which has been

189 previously been shown to elicit changes in heart rate and blood pressure (Heffernan et al. 2014).

Participant's identification accuracy was titrated to $60 \%$ in order to produce equivalent

191 hemodynamic responses across sea level and HA Stroop testing. This was achieved via manipulation of

192 the inter-item timing intervals (ITI; i.e. the time between presentation of each color-word challenge).

193 For every three consecutive items answered correctly the ITI was decreased by 300 ms (shortest ITI of

$194400 \mathrm{~ms}$ ). Similarly, three consecutive missed items would increase the ITI by 300 ms (longest ITI of 5,000 
$195 \mathrm{~ms})$. If the participant did not respond in time, a large "TOO LATE!" prompt was displayed before the 196 next item was displayed.

197

\section{Cerebral Blood Flow}

Middle cerebral artery (MCA) blood flow velocity was assessed using a 2-MHz transcranial Doppler (TCD) probe (DWL Doppler Box-X, Compumedics, Germany) applied to the left temporal window. Mean blood flow velocity (MnV) was measured at a depth of 50-65mm, as is commonly reported for MCA measurements (Xu et al. 2012). All repeated measurements within each participant were taken at the same depth and position to ensure recapture of the same cerebral artery. The envelope of the velocity spectrum and mean velocity was calculated by a standard algorithm implemented on the instrument with use of a fast Fourier transform. MCA blood flow velocity was also expressed relative to blood pressure by calculating MCA conductance as (MCA MnV/MAP) x 100, where MAP is mean arterial pressure calculated as $1 / 3$ systolic $+2 / 3$ diastolic blood pressure.

\section{Common Carotid Artery Blood Flow}

Images of the distal left common carotid artery (CCA) were obtained using Doppler ultrasound (ProSound $\alpha 7$, Aloka, Tokyo, Japan) and a 7.5-10.0 MHz linear-array probe. CCA diameters were measured from inside the near-wall intima-media to far-wall intima-media across a $5 \mathrm{~mm}$ region of interest via semi-automated digital calipers during systole and diastole (indicated by the R-wave and end of the T-wave from simultaneous ECG gating). Average diameter was calculated as (1/3 systolic diameter $+2 / 3$ diastolic diameter). Mean blood flow velocities $(\mathrm{MnV}$ ) were measured using Dopplerultrasound with an insonation angle $\leq 60^{\circ}$ for all measures and sample volume manually adjusted to encompass the entire vessel. Mean velocity was calculated as: $\mathrm{MnV}=\int \mathrm{V}(\mathrm{t}) \mathrm{dt} / \mathrm{FT}$, where $\int \mathrm{V}(\mathrm{t}) \mathrm{dt}$ is the velocity-time integral of the velocity waveform and FT is flow time. Velocity waveforms were measured. 
217 Blood flow was calculated as $\pi \times(1 / 3 \text { systolic radius }+2 / 3 \text { diastolic radius })^{2} \times M n V \times 60$. All images were

218 stored for later offline analysis by a single investigator who remained blinded regarding which treatment

219 contained nitrate.

\section{Brachial Blood Pressure}

Systolic blood pressure (SBP) and diastolic brachial blood pressure (DBP) were measured prior to

222 each set of vascular measures (at normoxia and hypoxia) using a validated, automated oscillometric cuff

223 (EW3109, Panasonic Electric Works, Secaucus NJ). Pressures were taken in duplicate and averaged. If

224 values were different by more than $5 \mathrm{mmHg}$ a third measure was obtained and the average of the 2

225 closest measures was used for subsequent analyses.

227 Global assessment of cognitive function was made through a validated computer-based program

228 (WebNeuro; Brain Resource, San Francisco, CA) which has sound test-retest reliability (Williams et al.

229 2005). Participants engaged in a series of cognitive tasks while sitting at a laptop computer with a

230 standard mouse/keyboard. The cognitive tests covered sensorimotor, memory, social cognition,

231 attention, and executive function domains and the specific tasks have been described in detail

232 elsewhere (Silverstein et al. 2007). Brief summaries of the tests are provided in Table 1.

\section{Statistical Analyses}

Effect size values were estimated from previous literature for cerebral blood flow (0.65)(Poulin

235 and Robbins 1996), reaction time (0.48)(Li et al. 2012) and memory (0.89)(Wang et al. 2013) in hypoxia,

236 identifying an average effect size of 0.67 . Therefore, for a power of 0.80 with alpha set as 0.05 for a two-

237 tailed t-test, approximately 20 participants was determined to be sufficient to observe similar changes in 238 blood flow and cognitive function during hypoxia. 
All data are presented as mean \pm standard deviation. All data was normally distributed, as tested using histograms, Q-Q plots, and Shapiro-Wilk tests, thus no variables were logarithmically transformed. The effect of altitude exposure was tested using paired t-tests between normoxia and

242 hypoxia. The effect of NIT was tested using paired t-tests between NIT and PLA in hypoxia. NVC was 243 assessed using absolute $\Delta$ scores, calculated as cognitive engagement values - baseline values, for each treatment (PLA, NIT) and condition (normoxia, hypoxia). A one-sample t-test compared NVC change scores to zero to determine if there was significant hemodynamic coupling during cognitive activity.

246 Significance was set a priori at $\mathrm{p}<0.05$.

\section{Results}

oxygen in the hypoxic chamber $(11.6 \pm 0.1 \%, \mathrm{PLA} ; 11.7 \pm 0.1 \%, \mathrm{NIT} ; \mathrm{p}=0.34)$ were not significantly different between treatments. Hypoxia resulted in similar significant decreases in $\mathrm{SaO}_{2}(98 \pm 2$ vs $75 \pm$

$6 \%$ PLA; $98 \pm 2$ vs $75 \pm 7 \%$ NIT; p < 0.001) and ET-CO $(39 \pm 2$ vs $33 \pm 2$ mmHg PLA; $39 \pm 3$ vs $34 \pm 2$ mmHg

252 NIT; $p<0.001$ ) in both treatments compared to normoxia. Salivary nitrite was similar between

253 treatments under normoxic conditions (0.1 $\pm 0.2 \mathrm{AU}$ PLA, $0.1 \pm 0.2 \mathrm{AU}$ NIT; $p=0.49)$ prior to NIT/PLA

254 consumption. Following acute supplementation, salivary nitrite was significantly greater in hypoxia for 255 NIT $(2.7 \pm 1.0 \mathrm{AU})$ compared to PLA $(0.2 \pm 0.3 \mathrm{AU} ; \mathrm{p}<0.001)$. 
262 there was no differences in cerebrovascular measures between PLA and NIT under hypoxic exposure ( $p$

$263>0.05)$, indicating NIT supplementation did not alter resting vascular responses to hypoxia.

Effect of hypoxia on neurovascular coupling

There were increases in MCA mean flow velocity during Stroop task compared to baseline in

266 both treatments and conditions, providing evidence of NVC $(p<0.05$; Table 3). For both PLA and NIT

267 treatments, increase in MCA flow velocity during Stroop ( $\Delta \mathrm{mV}$; Table 2$)$ was not different in hypoxia

268 compared to normoxia $(p>0.05)$. MCA conductance was unaltered during cognitive activity in both

269 hypoxia and normoxia ( $p>0.05)$. CCA diameter increased during Stroop compared to resting baseline in

270 normoxia $(p<0.01)$. CCA diameter change during Stroop was attenuated in both PLA and NIT treatments

271 in hypoxia compared to normoxia $(p<0.05)$. Despite reductions in CCA vasodilation, blood flow during

272 Stroop was ultimately similar between hypoxia and normoxia within both treatments $(p<0.05)$ which

273 may have resulted from a tendency for attenuated reductions in CCA mean velocity during Stroop in

274 hypoxia $(p=0.062)$. Overall, there were no differences in cerebrovascular response to Stroop in hypoxia

275 between NIT and PLA treatments $(p>0.05)$, indicating NIT did not significantly alter NVC under hypoxic

276 conditions compared to PLA.

\section{Effect of hypoxia on cognitive function}

278 There were no differences in cognitive function at normoxic baseline between PLA and NIT treatments

279 ( $p>0.05)$. A significant effect of hypoxia was detected within the memory and information processing

280 domains (Table 4). Memory recognition was lower in hypoxia compared to normoxia in both PLA and

281 NIT treatments $(p<0.05)$. This was driven by greater intrusion errors and lower memory recognition

282 performance in both PLA and NIT treatments in hypoxia $(p<0.05)$. Accuracy and RT during the visual

283 interference task was improved in both PLA and NIT treatments in hypoxia compared to normoxia $(p<$

284 0.05). Emotion recognition index was lower in both PLA and NIT treatments in hypoxia compared to 
285

286

287

288

289

290

291

292

293

294

295

296

297

298

299

300

301

302

303

normoxia $(p<0.05)$. Cognitive performance in the remaining cognitive domains were not affected by hypoxic exposure in either PLA or NIT treatments $(p>0.05)$. The reductions in aforementioned domains of cognitive function in hypoxia were similar between PLA and NIT treatments ( $p>0.05$ for interaction), indicating NIT supplementation was not effective in altering cognitive function during hypoxic exposure. There were no changes in any other cognitive domain from normoxia to hypoxia with either PLA or NIT $(p>0.05)$.

\section{Discussion}

This study investigated the effect of acute dietary nitrate supplementation in the form of beetroot juice on NVC and cognitive function under hypoxic conditions. The overarching hypothesis was that nitrate supplementation would result in greater NO formation and act to increase vasodilation, blood flow, and oxygen delivery to the cerebrovasculature, thereby optimizing NVC and improving cognitive performance following rapid exposure to systemic hypoxia. The primary findings of the study were as follows: 1) NVC (assessed as the increase in MCA mean flow velocity during cognitive engagement) was similar in normoxia versus hypoxia; 2) while aspects of memory performance were reduced during hypoxia, other aspects of cognitive performance (executive function, attention, sensorimotor processing) were preserved; 3) nitrate did not alter cognitive performance nor did it alter cerebral blood flow during cognitive engagement (NVC) in hypoxia. Thus, acute dietary nitrate supplementation does not affect NVC or cognitive performance under hypoxic conditions in young, healthy men.

Acute exposure to systemic hypoxia results in complex alterations in neurophysiological and psychological function. Examining the impact of nitrate supplementation on NVC and cognitive function during hypoxia has important implications for populations regularly exposed to hypoxic conditions such as (mountaineers (Merz et al. 2013), aircraft pilots (Petrassi et al. 2012), and military personnel (Adam 
et al. 2008)), and to clinical populations that suffer acute ischemic events (Anderson and Arciniegas

2010; Peskine et al. 2010). Under normoxic conditions, short-term nitrate loading (1-3 days) has been reported to increase regional cerebral perfusion in older adults (Presley et al. 2011), and reduce

311 hyperemic lag times during visual stimulation (Aamand et al. 2013), indicative of improved NVC.

312 Additionally, normoxic data would suggest that nitrate supplementation can increase brain perfusion

313 (Presley et al. 2011), although this is not a universal finding (Aamand et al. 2013), and acutely improve

314 cognitive function during a mental fatigue protocol (Wightman et al. 2015). With this information as our

315 foundation, we set out to explore the effect of acute nitrate supplementation on NVC and cognitive

316 function in systemic hypoxia.

Oxygen tension is a fundamental factor in determining blood flow to target organs such as skeletal muscle or the brain. Hypoxic conditions result in a compensatory hyperemic response (Jia et al. 2011 ) in order to offset reductions in arterial oxygen content. We noted significant increases in CCA diameter, CCA flow and MCA flow velocity with hypoxia. Nearly $80 \%$ of CCA blood flow feeds the internal carotid, which evolves into the MCA and provides approximately $80 \%$ of the blood supply to the

322 brain (Poulin and Robbins 1996; Farkas and Luiten 2001). Thus some intracranial flow regulation may 323 occur extracranially (Willie et al. 2014). Our findings are consistent with recent comprehensive studies by Lewis et al. (2014) who reported significant increases in CCA dilation following slightly more prolonged exposure (72-96 hr) after both normobaric (Lewis et al. 2014b) and hypobaric hypoxia (Lewis et al. 2014a). Moreover, NVC was largely maintained during hypoxia in the current study. Thus, there

327 were additional increases in carotid and cerebral flow during cognitive engagement above levels initially 328 caused by hypoxia. Maintenance of NVC occurred despite an attenuated CCA dilatory response during 329 cognitive engagement in hypoxia. Total inflow volume is ultimately determined by changes in vessel 330 diameter and flow velocity. It is possible that the CCA was maximally dilated under the current hypoxic 331 stimuli, resulting in cognitive activity-dependent hyperemia achieved through alteration of blood flow 
332 velocity rather than diameter, although future studies should directly interrogate this observation using 333 endothelium-independent dilators (i.e. sodium nitroprusside).

The current study observed significant decreases in memory function following approximately

2.5 hours of hypoxia. Specifically, there were reductions in verbal memory and intrusion indices, resulting from decrements in immediate and delayed memory accuracy and greater error rates,

337 respectively. These decrements in the memory domain are consistent with previous reports (Dykiert et 338 al. 2010; Li et al. 2012; Malle et al. 2013; Wang et al. 2013) across a variety of hypoxic stimuli ranging 339 from 2,800m (Wu et al. 2002) to 9,449m (Malle et al. 2013). There was also significant dysfunction with 340 regards to emotion recognition. Emotion recognition in this study was assessed as 1) the ability to 341 correctly identify emotion based on facial expressions and 2 ) the ability to recall ( $\approx 10$ minutes later) 342 which facial expressions had been previously presented. Thus, it is possible that the reductions in 343 emotion recognition observed herein may be partially related to/driven by impaired memory function 344 that potentially impacted the emotion recall performance. We noted no significant changes for the 345 remaining cognitive domains. Indeed, some domains of cognitive function may not be affected by 346 hypoxia. Performance on simple tasks, such as 2-choice RT and finger tapping, is maintained at altitudes 347 below 6,000m (Virues-Ortega et al. 2004; Petrassi et al. 2012). Consistent with these reports, we 348 observed no significant changes in finger tapping speed, choice RT, or go-no-go tasks. We also observed 349 no effect of hypoxia on verbal learning rate and executive function and this too is in line with previous 350 reports (Paul and Fraser 1994; Asmaro et al. 2013). This supports a recent review (Petrassi et al. 2012) 351 suggesting that some domains may not be sensitive to acute hypoxia (Pavlicek et al. 2005; Petrassi et al. 352 2012), or perhaps require longer exposure or more extreme hypoxia to observe effects. 
and nitrate (Muggeridge et al. 2013b) and affect cognitive function in normoxia (Wightman et al. 2015),

356 although this positive effect on cognition is not a universal finding (Kelly et al. 2013; Thompson et al.

357 2014). Further increasing the dose in order to maximize effects may not be efficacious since nitrate

358 loading ( $\approx 50 \mathrm{mmol} / \mathrm{day})$ does not increase cerebral oxygenation in hypoxia (Masschelein et al. 2012) and

359 supra-physiological concentrations of nitrite have been shown to impair NVC by increasing local resting

360 cerebral flow, thus limiting cerebrovascular reserve in response to neuronal activation (Piknova et al.

361 2011). Nitrite supplementation has been shown to increase NVC in animal models, but only after

362 inhibition of normal NO production within the brain (Piknova et al. 2011). Thus, nitrate

363 supplementation in our study may not have altered hypoxic vasodilation and NVC because our young

364 participants did not have impaired NO-bioavailability under hypoxic conditions. Ultimately, the

365 mechanisms responsible for hypoxia-induced cerebrovascular vasodilation and hyperemia may be multi-

366 factorial and rely on multiple, redundant pathways similar to those observed with the peripheral

367 vasodilatory responses to exercise (Joyner and Casey 2009). A recent review has suggested these

368 cerebral mechanisms may not only involve NO, but also several other factors such as adenosine,

369 prostaglandins, $\mathrm{PaO}_{2}$ and anaerobic neuronal metabolism (Willie et al. 2014). Moreover, there may also

370 be competing neural mechanisms related to regional release of neurotransmitters/neuropeptides (i.e.

371 brain-derived neurotrophic factor, vasopressin, neuropeptide $Y$, dehydroepiandrosterone etc) along

372 with disparate changes in oxygen extraction at the tissue level.

Preservation of NVC in hypoxia may thusly preserve a majority of cognitive functions as seen

374 herein. The question then arises: why were some cognitive functions conserved while others

375 detrimentally impacted? Domains related to executive function, reaction time, attention,

376 concentration, and information processing (arguably considered more vital for survival in a fight-or-flight

377 situation) are largely under prefrontal cortex regulation while domains such as verbal memory and

378 emotion recognition memory may be of hippocampal origin. The hippocampus has been found to be 
379

380

381

382

383

384

385

386

more sensitive to hypoxia-mediated oxidative stress and subsequent hypoxic-ischemic injury than the cortex (Maiti et al. 2006; Hota et al. 2007; Maiti et al. 2008), which has been linked to heterogeneity in regional hemodynamic responsiveness to hypoxia (Dunn et al. 1999). Moreover, these hypoxic alterations in hemodynamics and oxidative stress in the hippocampus have been specifically linked to impairments in memory (Maiti et al. 2008). Future studies should explore both anterior cerebral arteries (frontal cortex) and posterior cerebral arteries (hippocampus) as part of NVC protocols that explore cognitive function in hypoxia.

\section{Limitations and future directions}

The participants in the current study were generally young ( $23 \pm 3 \mathrm{yrs}$ ), healthy males which limits applicability to other populations. Results may differ if this study was conducted in females based on hormonal differences introduced by the menstrual cycle and the subsequent timing of measures during the cycle. Investigating the effects of hypoxia on the cognitive and cerebrovascular function is important across a large range of ages because vascular responses to hypoxia may change with age (Petrassi et al. 2012). Older adults or those with multiple cardiovascular risk factors have been reported to experience neurovascular uncoupling (D'Esposito et al. 1999; Stroobant and Vingerhoets 2000; Groschel et al. 2007) and suffer from impaired sympatholysis (Dinenno et al. 2005; Casey et al. 2014) which is of particular importance since a portion of hypoxic hyperemia is due to sympatholysis overriding sympathetic-mediated constriction (Dinenno et al. 2003). Studying the acute effect of nitrate ingestion on NVC in hypoxia in this population may be beneficial as nitrate has been shown to restore sympatholysis in older adults during hypoxic exercise (Casey et al. 2014) and alter NVC in older adults (Presley et al. 2011). It is possible that individuals repeatedly exposed to subacute hypoxia/ischemia may respond differently to nitrate supplementation due to hypoxic preconditioning. Studies suggest that repeated subacute hypoxic exposure may offer neurovascular protection (Poinsatte et al. 2015), 
402 stimulate cerebrovascular remodeling (Boroujerdi and Milner 2015), and may improve oxygen

403 saturation (Foster et al. 2014) and abrogate oxidative stress (Berger et al. 2015) during additional

404 ischemic events. Whether hypoxic preconditioning alters the effect of nitrate on NVC and cognitive

405 function in hypoxia was beyond the scope of the current study and requires future research.

Cerebral blood flow is dependent on arterial gases, blood pressure, and neural activity (Willie et

407 al. 2014). Hypoxia is accompanied by a hyperventilatory response that, although intended to defend

408 against arterial hypoxemia, may have a profound effect on the cerebrovasculature via hyperventilation-

409 induced hypocapnic vasoconstriction (Brugniaux et al. 2007). In the current study, participants were

410 exposed to similar hypoxic stimuli (severity and duration), and demonstrated similar acute

411 hyperventilation responses to hypoxia (evident by similar reductions in $\mathrm{ET}-\mathrm{CO}_{2}$ ), thus we do not believe

412 the differences in ventilatory responses to hypoxia altered the cerebrovascular responses between

413 treatments. Mean arterial pressure was not significantly different between conditions or treatments,

414 however autoregulation may be compromised in hypoxia (Nishimura et al. 2010) although this is

415 somewhat debated (Querido et al. 2013). Cerebral perfusion becomes more dependent on pressure in

416 the presence of impaired cerebral autoregulation. Thus, small changes in pressure may influence NVC.

417 Indeed, when cerebral blood flow velocity was expressed as conductance (i.e flow relative to pressure)

418 we noted no significant NVC, suggesting that the increases in MCA mean velocity during cognitive

419 activity may be the result of a combination of blood pressure-mediated effects of mental stress and

420 neural activity. Future research is required to further tease out the independent and combined roles of

$421 \mathrm{CO}_{2}$, blood pressure/autoregulation, and neural activity on cerebral hemodynamics under hypoxic

422 conditions.

423 Conclusions 
424 Our novel assessment of NVC revealed a preservation of carotid and cerebral flow during 425 cognitive engagement in hypoxia that may be related to preferential conservation of select cognitive 426 domains (executive function, reaction time, attention and concentration, information processing) but 427 not others (memory and emotion and recognition). Ultimately, we found that compared to placebo, 428 acute nitrate supplementation did not affect NVC during hypoxic exposure nor did it alter cognitive 429 performance in young healthy men.

430 Acknowledgments The authors have no conflicts of interest to disclose. This research was supported by an ACSM

432 Foundation Research Grant from the American College of Sports Medicine Foundation. 
Aamand, R., Dalsgaard, T., Ho, Y.C., Moller, A., Roepstorff, A., andLund, T.E. 2013. A NO way to BOLD?: Dietary nitrate alters the hemodynamic response to visual stimulation. Neuroimage 83C: 397-407. Adam, G.E., Fulco, C.S., andMuza, S.R. 2008. Multitask performance at sea level and high altitude. Proceedings of the 25th Army Science Conference. Anderson, C.A. and Arciniegas, D.B. 2010. Cognitive sequelae of hypoxic-ischemic brain injury: a review. NeuroRehabilitation 26: 47-63. processes under hypoxic conditions. Aviat Space Environ Med 84: 1159-65. Attwell, D., Buchan, A.M., Charpak, S., Lauritzen, M., Macvicar, B.A., andNewman, E.A. 2010. Glial and neuronal control of brain blood flow. Nature 468: 232-43. Babbar, R. and Agarwal, S. 2012. A new approach to hypobaric hypoxia induced cognitive impairment. Indian J Med Res 136: 365-7. studies. BMC Neurol 14: 43.

Berger, M.M., Kohne, H., Hotz, L., Hammer, M., Schommer, K., Bartsch, P., andMairbaurl, H. 2015. Remote ischemic preconditioning delays the onset of acute mountain sickness in normobaric hypoxia. Physiol Rep 3.

Boroujerdi, A. and Milner, R. 2015. Defining the critical hypoxic threshold that promotes vascular remodeling in the brain. Exp Neurol 263: 132-40.

Brugniaux, J.V., Hodges, A.N., Hanly, P.J., andPoulin, M.J. 2007. Cerebrovascular responses to altitude. Respir Physiol Neurobiol 158: 212-23.

Casey, D.P. and Joyner, M.J. 2011. Local control of skeletal muscle blood flow during exercise: influence of available oxygen. J Appl Physiol (1985) 111: 1527-38. Supplementation Enhances Compensatory Vasodilation during Hypoxic Exercise in Older Adults. J Appl Physiol (1985): jap.00662.2014.

Chen, C.C., Chen, Y.C., Hsiao, H.Y., Chang, C., andChern, Y. 2013. Neurovascular abnormalities in brain disorders: highlights with angiogenesis and magnetic resonance imaging studies. J Biomed Sci 20: 47. D'Esposito, M., Zarahn, E., Aguirre, G.K., andRypma, B. 1999. The effect of normal aging on the coupling of neural activity to the bold hemodynamic response. Neuroimage 10: 6-14.

Dinenno, F.A., Joyner, M.J., andHalliwill, J.R. 2003. Failure of systemic hypoxia to blunt alpha-adrenergic vasoconstriction in the human forearm. J Physiol 549: 985-94.

Dinenno, F.A., Masuki, S., andJoyner, M.J. 2005. Impaired modulation of sympathetic alpha-adrenergic vasoconstriction in contracting forearm muscle of ageing men. J Physiol 567: 311-21.

Dunn, J.F., Wadghiri, Y.Z., andMeyerand, M.E. 1999. Regional heterogeneity in the brain's response to hypoxia measured using BOLD MR imaging. Magn Reson Med 41: 850-4.

Duplain, H., Sartori, C., Lepori, M., Egli, M., Allemann, Y., Nicod, P., andScherrer, U. 2000. Exhaled nitric oxide in high-altitude pulmonary edema: role in the regulation of pulmonary vascular tone and evidence for a role against inflammation. Am J Respir Crit Care Med 162: 221-4.

Dykiert, D., Hall, D., van Gemeren, N., Benson, R., Der, G., Starr, J.M., andDeary, I.J. 2010. The effects of high altitude on choice reaction time mean and intra-individual variability: Results of the Edinburgh Altitude Research Expedition of 2008. Neuropsychology 24: 391-401.

Fan, J.L., Burgess, K.R., Basnyat, R., Thomas, K.N., Peebles, K.C., Lucas, S.J., Lucas, R.A., Donnelly, J., Cotter, J.D., andAinslie, P.N. 2010. Influence of high altitude on cerebrovascular and ventilatory responsiveness to CO2. J Physiol 588: 539-49. 
Farkas, E. and Luiten, P.G. 2001. Cerebral microvascular pathology in aging and Alzheimer's disease. Prog Neurobiol 64: 575-611. Foster, G.P., Giri, P.C., Rogers, D.M., Larson, S.R., andAnholm, J.D. 2014. Ischemic preconditioning improves oxygen saturation and attenuates hypoxic pulmonary vasoconstriction at high altitude. High Alt Med Biol 15: 155-61. Groschel, K., Terborg, C., Schnaudigel, S., Ringer, T., Riecker, A., Witte, O.W., andKastrup, A. 2007. Effects of physiological aging and cerebrovascular risk factors on the hemodynamic response to brain activation: a functional transcranial Doppler study. Eur J Neurol 14: 125-31. Heffernan, K.S., Spartano, N.L., Augustine, J.A., Lefferts, W.K., Hughes, W.E., Mitchell, G.F., Jorgensen, R.S., andGump, B.B. 2014. Carotid Artery Stiffness and Hemodynamic Pulsatility During Cognitive Engagement in Healthy Adults: A Pilot Investigation. Am J Hypertens. Hord, N.G., Tang, Y., andBryan, N.S. 2009. Food sources of nitrates and nitrites: the physiologic context for potential health benefits. Am J Clin Nutr 90: 1-10. Hota, S.K., Barhwal, K., Singh, S.B., andllavazhagan, G. 2007. Differential temporal response of hippocampus, cortex and cerebellum to hypobaric hypoxia: a biochemical approach. Neurochem Int 51: 384-90.

ladecola, C. 2004. Neurovascular regulation in the normal brain and in Alzheimer's disease. Nat Rev Neurosci 5: 347-60.

Jia, Y., Li, P., Dziennis, S., andWang, R.K. 2011. Responses of peripheral blood flow to acute hypoxia and hyperoxia as measured by optical microangiography. PLoS One 6: e26802.

Joyner, M.J. and Casey, D.P. 2009. The catecholamines strike back. What NO does not do. Circ J 73: 1783-92.

Kelly, J., Fulford, J., Vanhatalo, A., Blackwell, J.R., French, O., Bailey, S.J., Gilchrist, M., Winyard, P.G., andJones, A.M. 2013. Effects of short-term dietary nitrate supplementation on blood pressure, $\mathrm{O} 2$ uptake kinetics, and muscle and cognitive function in older adults. Am J Physiol Regul Integr Comp Physiol 304: R73-83.

Levett, D.Z., Fernandez, B.O., Riley, H.L., Martin, D.S., Mitchell, K., Leckstrom, C.A., Ince, C., Whipp, B.J., Mythen, M.G., Montgomery, H.E., Grocott, M.P., Feelisch, M., andCaudwell Extreme Everest Research, G. 2011. The role of nitrogen oxides in human adaptation to hypoxia. Sci Rep 1: 109. Lewis, N.C., Bailey, D.M., Dumanoir, G.R., Messinger, L., Lucas, S.J., Cotter, J.D., Donnelly, J., McEneny, J., Young, I.S., Stembridge, M., Burgess, K.R., Basnet, A.S., andAinslie, P.N. 2014a. Conduit artery structure and function in lowlanders and native highlanders: relationships with oxidative stress and role of sympathoexcitation. J Physiol 592: 1009-24.

Lewis, N.C., Messinger, L., Monteleone, B., andAinslie, P.N. 2014b. Effect of acute hypoxia on regional cerebral blood flow: effect of sympathetic nerve activity. J Appl Physiol (1985) 116: 1189-96. Li, P., Zhang, G., You, H.Y., Zheng, R., andGao, Y.Q. 2012. Training-dependent cognitive advantage is suppressed at high altitude. Physiol Behav 106: 439-45.

Lundberg, J.O., Weitzberg, E., andGladwin, M.T. 2008. The nitrate-nitrite-nitric oxide pathway in physiology and therapeutics. Nat Rev Drug Discov 7: 156-67.

Lupo, M., Troisi, E., Chiricozzi, F.R., Clausi, S., Molinari, M., andLeggio, M. 2015. Inability to Process Negative Emotions in Cerebellar Damage: a Functional Transcranial Doppler Sonographic Study. Cerebellum.

Maiti, P., Singh, S.B., Mallick, B., Muthuraju, S., andllavazhagan, G. 2008. High altitude memory impairment is due to neuronal apoptosis in hippocampus, cortex and striatum. J Chem Neuroanat 36: 227-38.

526 hypoxia induces oxidative stress in rat brain. Neurochem Int 49: 709-16. 
Malle, C., Quinette, P., Laisney, M., Bourrilhon, C., Boissin, J., Desgranges, B., Eustache, F., andPierard, C. 2013. Working memory impairment in pilots exposed to acute hypobaric hypoxia. Aviat Space Environ Med 84: 773-9. Masschelein, E., Van Thienen, R., Wang, X., Van Schepdael, A., Thomis, M., andHespel, P. 2012. Dietary nitrate improves muscle but not cerebral oxygenation status during exercise in hypoxia. J Appl Physiol 113: 736-45. Merz, T.M., Bosch, M.M., Barthelmes, D., Pichler, J., Hefti, U., Schmitt, K.U., Bloch, K.E., Schoch, O.D., Hess, T., Turk, A.J., andSchwarz, U. 2013. Cognitive performance in high-altitude climbers: a comparative study of saccadic eye movements and neuropsychological tests. Eur J Appl Physiol 113: 2025-37. Muggeridge, D.J., Howe, C.C., Spendiff, O., Pedlar, C., James, P.E., andEaston, C. 2013a. The effects of a single dose of concentrated beetroot juice on performance in trained flatwater kayakers. Int J Sport Nutr Exerc Metab 23: 498-506. Muggeridge, D.J., Howe, C.C., Spendiff, O., Pedlar, C., James, P.E., andEaston, C. 2013b. A Single Dose of Beetroot Juice Enhances Cycling Performance in Simulated Altitude. Med Sci Sports Exerc. Nichols, W.W. and O'Rourke, M.F. 2005. McDonald's blood flow in arteries. Theoretical, experimental and clinical principles. London: Hodder Arnold. Nishimura, N., Iwasaki, K., Ogawa, Y., andAoki, K. 2010. Decreased steady-state cerebral blood flow velocity and altered dynamic cerebral autoregulation during 5-h sustained 15\% O2 hypoxia. J Appl Physiol (1985) 108: 1154-61.

Paul, M.A. and Fraser, W.D. 1994. Performance during mild acute hypoxia. Aviat Space Environ Med 65: 891-9.

Pavlicek, V., Schirlo, C., Nebel, A., Regard, M., Koller, E.A., andBrugger, P. 2005. Cognitive and emotional processing at high altitude. Aviat Space Environ Med 76: 28-33.

550 Payne, H., Gutierrez-Sigut, E., Subik, J., Woll, B., andMacSweeney, M. 2015. Stimulus rate increases sonography. Neuropsychologia 72: 59-69.

Peskine, A., Rosso, C., Picq, C., Caron, E., andPradat-Diehl, P. 2010. Neurological sequelae after cerebral anoxia. Brain Inj 24: 755-61.

Petrassi, F.A., Hodkinson, P.D., Walters, P.L., andGaydos, S.J. 2012. Hypoxic hypoxia at moderate altitudes: review of the state of the science. Aviat Space Environ Med 83: 975-84.

Piknova, B., Kocharyan, A., Schechter, A.N., andSilva, A.C. 2011. The role of nitrite in neurovascular coupling. Brain Res 1407: 62-8.

Poinsatte, K., Selvaraj, U.M., Ortega, S.B., Plautz, E.J., Kong, X., Gidday, J.M., andStowe, A.M. 2015. Quantification of neurovascular protection following repetitive hypoxic preconditioning and transient middle cerebral artery occlusion in mice. J Vis Exp: e52675.

Poulin, M.J. and Robbins, P.A. 1996. Indexes of flow and cross-sectional area of the middle cerebral artery using doppler ultrasound during hypoxia and hypercapnia in humans. Stroke 27: 2244-50. Presley, T.D., Morgan, A.R., Bechtold, E., Clodfelter, W., Dove, R.W., Jennings, J.M., Kraft, R.A., King, S.B., Laurienti, P.J., Rejeski, W.J., Burdette, J.H., Kim-Shapiro, D.B., andMiller, G.D. 2011. Acute effect of a high nitrate diet on brain perfusion in older adults. Nitric Oxide 24: 34-42.

Querido, J.S., Ainslie, P.N., Foster, G.E., Henderson, W.R., Halliwill, J.R., Ayas, N.T., andSheel, A.W. 2013. Dynamic cerebral autoregulation during and following acute hypoxia: role of carbon dioxide. J Appl Physiol (1985) 114: 1183-90.

Silverstein, S.M., Berten, S., Olson, P., Paul, R., Willams, L.M., Cooper, N., andGordon, E. 2007. Development and validation of a World-Wide-Web-based neurocognitive assessment battery: WebNeuro. Behav Res Methods 39: 940-9.

Sorond, F.A., Hurwitz, S., Salat, D.H., Greve, D.N., andFisher, N.D. 2013. Neurovascular coupling, cerebral white matter integrity, and response to cocoa in older people. Neurology 81: 904-9. 
575

576

577

578

579

580

581

582

583

584

585

586

587

588

589

590

591

592

593

594

595

596

597

598

599

600

601

602

603
Stroobant, N. and Vingerhoets, G. 2000. Transcranial Doppler ultrasonography monitoring of cerebral hemodynamics during performance of cognitive tasks: a review. Neuropsychol Rev 10: 213-31.

Thompson, K.G., Turner, L., Prichard, J., Dodd, F., Kennedy, D.O., Haskell, C., Blackwell, J.R., andJones, A.M. 2014. Influence of dietary nitrate supplementation on physiological and cognitive responses to incremental cycle exercise. Respir Physiol Neurobiol 193: 11-20.

Van Mil, A.H., Spilt, A., Van Buchem, M.A., Bollen, E.L., Teppema, L., Westendorp, R.G., andBlauw, G.J. 2002. Nitric oxide mediates hypoxia-induced cerebral vasodilation in humans. J Appl Physiol 92: 962-6. Vingerhoets, G. and Stroobant, N. 1999. Lateralization of cerebral blood flow velocity changes during cognitive tasks. A simultaneous bilateral transcranial Doppler study. Stroke 30: 2152-8.

Virues-Ortega, J., Buela-Casal, G., Garrido, E., andAlcazar, B. 2004. Neuropsychological functioning associated with high-altitude exposure. Neuropsychol Rev 14: 197-224.

Wang, J., Ke, T., Zhang, X., Chen, Y., Liu, M., Chen, J., andLuo, W. 2013. Effects of acetazolamide on cognitive performance during high-altitude exposure. Neurotoxicol Teratol 35: 28-33.

Wightman, E.L., Haskell-Ramsay, C.F., Thompson, K., Blackwell, J.R., Winyard, P.G., Forster, J., Jones, A.M., andKennedy, D.O. 2015. Dietary nitrate modulates cerebral blood flow parameters and cognitive performance in humans: a double-blind, placebo-controlled, crossover investigation. Physiol Behav. Williams, L.M., Simms, E., Clark, C.R., Paul, R.H., Rowe, D., andGordon, E. 2005. The test-retest reliability of a standardized neurocognitive and neurophysiological test battery: "neuromarker". Int J Neurosci 115: 1605-30.

Willie, C.K., Tzeng, Y.C., Fisher, J.A., andAinslie, P.N. 2014. Integrative regulation of human brain blood flow. J Physiol 592: 841-59.

Wolf, M.E. 2015. Functional TCD: regulation of cerebral hemodynamics--cerebral autoregulation, vasomotor reactivity, and neurovascular coupling. Front Neurol Neurosci 36: 40-56.

Wu, X.Y., Li, X.Y., Wang, J.T., Zhuang, Y., andDu, J.Y. 2002. Effects of simulated high altitude hypoxia on cognitive performance. Zhongguo Ying Yong Sheng Li Xue Za Zhi 18: 34-7.

Xu, T.Y., Staessen, J.A., Wei, F.F., Xu, J., Li, F.H., Fan, W.X., Gao, P.J., Wang, J.G., andLi, Y. 2012. Blood flow pattern in the middle cerebral artery in relation to indices of arterial stiffness in the systemic circulation. Am J Hypertens 25: 319-24. 
Table 1: Computerized cognitive battery task description.

\begin{tabular}{|c|c|c|c|}
\hline Domain & Task & Description & Construct \\
\hline Memory & $\begin{array}{l}\text { Memory } \\
\text { Recognition }\end{array}$ & $\begin{array}{l}\text { Presented with } 20 \text { words during } 4 \text { memorization trials. Then } \\
\text { presented with } 1 \text { target word and } 2 \text { distracter words, must } \\
\text { correctly identify word from memorization lists. } \approx 10 \text { minutes } \\
\text { later, memory recognition trial was repeated }\end{array}$ & $\begin{array}{l}\text { Immediate, } \\
\text { Delayed- } \\
\text { Recognition } \\
\text { Immediate, } \\
\text { Delayed Intrusions }\end{array}$ \\
\hline \multirow[t]{2}{*}{ Attention } & $\begin{array}{l}\text { Continuous } \\
\text { Performance } \\
\text { Test }\end{array}$ & $\begin{array}{l}\text { Presented with series of letters, must respond when the same } \\
\text { letter is presented twice in a row }\end{array}$ & $\begin{array}{l}\text { RT } \\
\text { Errors }\end{array}$ \\
\hline & $\begin{array}{l}\text { Forward } \\
\text { Digit Span }\end{array}$ & $\begin{array}{l}\text { Presented with series of digits, must correctly enter digits in } \\
\text { the order they were presented. Number of digits increased } \\
\text { sequentially from 3-7 }\end{array}$ & $\begin{array}{l}\text { Recall Span } \\
\text { Trials Correct }\end{array}$ \\
\hline \multirow[t]{5}{*}{$\begin{array}{l}\text { Executive } \\
\text { Function }\end{array}$} & $\begin{array}{l}\text { Switching of } \\
\text { Attention }\end{array}$ & $\begin{array}{l}\text { Similar to Trials B task. Presented with series of letters and } \\
\text { numbers, must click on numbers and letters in alternating } \\
\text { ascending order (1, A, 2, B etc.) as fast as possible }\end{array}$ & $\begin{array}{l}\text { Duration } \\
\text { RT } \\
\text { Accuracy (\%) }\end{array}$ \\
\hline & $\begin{array}{l}\text { Visual } \\
\text { Interference }\end{array}$ & $\begin{array}{l}\text { Presented with target word (e.g. "red"), must identify what } \\
\text { color the word says while ignoring the color of the paint as } \\
\text { fast as possible. }\end{array}$ & $\begin{array}{l}\text { Correct trials } \\
\text { Errors } \\
\text { RT }\end{array}$ \\
\hline & $\begin{array}{l}\text { Verbal } \\
\text { Interference }\end{array}$ & $\begin{array}{l}\text { Presented with target word (e.g. "red"), must identify what } \\
\text { color the word is painted while ignoring the color the word } \\
\text { says as fast as possible. }\end{array}$ & $\begin{array}{l}\text { Correct trials } \\
\text { Errors } \\
\text { RT }\end{array}$ \\
\hline & Maze & $\begin{array}{l}\text { Presented with } 8 \times 8 \text { grid, must identify/learn the hidden path } \\
\text { through the grid, must complete twice without error }\end{array}$ & $\begin{array}{l}\text { Trials Completed } \\
\text { Path Learning Time } \\
\text { Accuracy (\%) } \\
\end{array}$ \\
\hline & Go/No-Go & $\begin{array}{l}\text { Must respond as quickly as possible when presented with } \\
\text { "go" stimuli, and must not respond when presented with "no- } \\
\text { go" stimuli }\end{array}$ & $\begin{array}{l}\text { RT } \\
\text { Errors }\end{array}$ \\
\hline \multirow[t]{2}{*}{$\begin{array}{l}\text { Sensori- } \\
\text { motor }\end{array}$} & $\begin{array}{l}\text { Motor } \\
\text { Tapping }\end{array}$ & Tap as fast as possible (30 sec) & Tapping Speed \\
\hline & Choice RT & Respond to 1 of 2 highlighted circle as fast as possible & Choice RT \\
\hline $\begin{array}{c}\text { Social } \\
\text { Cognition }\end{array}$ & $\begin{array}{l}\text { Emotion } \\
\text { Recognition }\end{array}$ & $\begin{array}{l}\text { Presented with faces of diverse emotional expression, must } \\
\text { correctly identify emotion out of } 6 \text { emotional words as quickly } \\
\text { as possible. } \approx 10 \text { minutes later, had to identify previously } \\
\text { viewed faces when presented next to distracter face as fast as } \\
\text { possible }\end{array}$ & $\begin{array}{l}\text { Correct (\%) } \\
\text { RT }\end{array}$ \\
\hline
\end{tabular}

RT, Reaction Time 
Table 2: Resting cerebrovascular measures across treatments and conditions (mean \pm SD)

\begin{tabular}{|c|c|c|c|c|}
\hline & \multicolumn{2}{|c|}{ Placebo } & \multicolumn{2}{|c|}{ Nitrate } \\
\hline & Normoxia & Hypoxia & Normoxia & Hypoxia \\
\hline Brachial MAP (mmHg) & $83 \pm 7$ & $87 \pm 6$ & $84 \pm 6$ & $85 \pm 9$ \\
\hline CCA Mean diameter (mm) & $5.91 \pm 0.46$ & $6.60 \pm 0.47^{*}$ & $5.75 \pm 0.48$ & $6.54 \pm 0.45^{*}$ \\
\hline CCA Mean velocity $\left(\mathrm{c} \cdot \mathrm{ms}^{-1}\right)$ & $37.2 \pm 4.6$ & $37.7 \pm 5.5$ & $38.0 \pm 3.9$ & $36.7 \pm 6.5$ \\
\hline CCA Blood flow $\left(\mathrm{ml} \cdot \mathrm{s}^{-1}\right)$ & $621.0 \pm 89.9$ & $786.5 \pm 139.7^{*}$ & $602.0 \pm 88.1$ & $746.5 \pm 126.3^{*}$ \\
\hline MCA Mean velocity $\left(\mathrm{m} \cdot \mathrm{s}^{-1}\right)$ & $66 \pm 15$ & $74 \pm 20^{*}$ & $68 \pm 16$ & $75 \pm 18^{*}$ \\
\hline MCA Conductance $\left(\mathrm{cm} \cdot \mathrm{s}^{-1} \cdot \mathrm{mmHg}^{-1}\right)$ & $80 \pm 19$ & $87 \pm 26$ & $82 \pm 19$ & $90 \pm 22$ \\
\hline Heart rate $\left(b \cdot \mathrm{min}^{-1}\right)$ & $56 \pm 9$ & $64 \pm 8^{*}$ & $54 \pm 7$ & $64 \pm 11^{*}$ \\
\hline $\mathrm{SaO}_{2}(\%)$ & $98 \pm 2$ & $75 \pm 6^{*}$ & $98 \pm 2$ & $75 \pm 7^{*}$ \\
\hline
\end{tabular}

$\mathrm{MAP}$, mean arterial pressure; CCA, common carotid artery; $\mathrm{MCA}$, middle cerebral artery; $\mathrm{SaO}_{2}$, arterial oxygen saturation.

$* p<0.05$ vs normoxia 
Table 3: Cardiovascular and cerebrovascular change values (cognitive engagement - rest) across treatments and conditions (mean \pm SD)

\begin{tabular}{|c|c|c|c|c|}
\hline & \multicolumn{2}{|c|}{ Placebo } & \multicolumn{2}{|c|}{ Nitrate } \\
\hline & Normoxia & Hypoxia & Normoxia & Hypoxia \\
\hline Brachial MAP (mmHg) & $9 \pm 6^{*}$ & $5 \pm 6^{*}$ & $4 \pm 4^{*}$ & $5 \pm 8^{*}$ \\
\hline CCA Mean diameter (mm) & $0.16 \pm 0.15^{*}$ & $0.00 \pm 0.20 \dagger$ & $0.17 \pm 0.19 *$ & $0.07 \pm 0.26+$ \\
\hline CCA Mean velocity $\left(\mathrm{cm} \cdot \mathrm{s}^{-1}\right)$ & $-0.8 \pm 3.1$ & $-0.4 \pm 3.6^{\wedge}$ & $-2.0 \pm 3.6^{*}$ & $0.5 \pm 3.1^{\wedge}$ \\
\hline CCA Blood flow $\left(\mathrm{ml} \cdot \mathrm{s}^{-1}\right)$ & $16.3 \pm 66.7$ & $-10.9 \pm 91.6$ & $-0.8 \pm 67.6$ & $28.3 \pm 90.2$ \\
\hline MCA Mean velocity $\left(m \cdot \mathrm{s}^{-1}\right)$ & $5 \pm 6^{*}$ & $5 \pm 9 *$ & $7 \pm 7^{*}$ & $6 \pm 7^{*}$ \\
\hline MCA Conductance $\left(\mathrm{cm} \cdot \mathrm{s}^{-1} \cdot \mathrm{mmHg}^{-1}\right)$ & $-2 \pm 8$ & $+1 \pm 10$ & $-4 \pm 10$ & $+2 \pm 12$ \\
\hline Heart rate $\left(b \cdot \mathrm{min}^{-1}\right)$ & $8 \pm 11^{*}$ & $7 \pm 8^{*}$ & $6 \pm 8^{*}$ & $5 \pm 7^{*}$ \\
\hline $\mathrm{SaO}_{2}(\%)$ & $0 \pm 2$ & $0 \pm 5$ & $0 \pm 2$ & $1 \pm 4$ \\
\hline
\end{tabular}

MAP, mean arterial pressure; CCA, common carotid artery; MCA, middle cerebral artery; $\mathrm{SaO}_{2}$, arterial oxygen saturation.

$* P<0.05$ vs zero; $\dagger p<0.05$ vs normoxia; ^trend $p=0.06$ vs normoxia 
Table 4: Cognitive performance and reaction times by cognitive domain across treatment and condition (mean \pm SD)

\begin{tabular}{|c|c|c|c|c|c|c|}
\hline \multirow[b]{2}{*}{ Domain } & \multirow[b]{2}{*}{ Task } & \multirow[b]{2}{*}{ Construct } & \multicolumn{2}{|c|}{ Placebo } & \multicolumn{2}{|c|}{ Nitrate } \\
\hline & & & Normoxia & Hypoxia & Normoxia & Hypoxia \\
\hline \multirow[t]{3}{*}{ Memory } & \multirow{3}{*}{$\begin{array}{l}\text { Memory } \\
\text { Recognition }\end{array}$} & Learning Rate & $0 \pm 1$ & $0 \pm 1$ & $0 \pm 1$ & $0 \pm 1$ \\
\hline & & Verbal Memory Index & $75 \pm 9$ & $70 \pm 10^{*}$ & $75 \pm 5$ & $71 \pm 10^{*}$ \\
\hline & & Verbal Intrusion Index & $5 \pm 9$ & $10 \pm 10 *$ & $5 \pm 5$ & $9 \pm 10^{*}$ \\
\hline \multirow{2}{*}{$\begin{array}{l}\text { Emotion } \\
\text { Identification }\end{array}$} & \multirow{2}{*}{$\begin{array}{l}\text { Emotion } \\
\text { Recognition }\end{array}$} & Emotion Recognition Index & $175 \pm 9$ & $173 \pm 11^{*}$ & $176 \pm 6$ & $170 \pm 11 *$ \\
\hline & & Average RT (ms) & $1671 \pm 318$ & $1591 \pm 518$ & $1620 \pm 281$ & $1520 \pm 256$ \\
\hline \multirow{2}{*}{$\begin{array}{l}\text { Working Memory } \\
\text { Capacity }\end{array}$} & \multirow{2}{*}{$\begin{array}{l}\text { Digit Span } \\
\text { (Forward) }\end{array}$} & Recall Span (forwards) & $7 \pm 1$ & $7 \pm 2$ & $7 \pm 1$ & $6 \pm 1$ \\
\hline & & Trials Correct (forwards) & $9 \pm 2$ & $9 \pm 3^{\wedge}$ & $9 \pm 2$ & $7 \pm 3^{\wedge}$ \\
\hline \multirow{8}{*}{$\begin{array}{l}\text { Information } \\
\text { Processing } \\
\text { Efficiency }\end{array}$} & \multirow[t]{2}{*}{ Visu-I (Word) } & \# Correct w/ Vis-I & $17 \pm 5$ & $20 \pm 4^{*}$ & $17 \pm 5$ & $19 \pm 5^{*}$ \\
\hline & & RT w/ Vis-I (ms) & $1186 \pm 321$ & $969 \pm 156^{*}$ & $1108 \pm 214$ & $1056 \pm 306^{*}$ \\
\hline & \multirow[t]{2}{*}{ Verb-I (Color) } & \# Correct w/ Ver-I & $17 \pm 4$ & $17 \pm 3$ & $18 \pm 5$ & $17 \pm 5$ \\
\hline & & RT w/ Ver-I (ms) & $1156 \pm 278$ & $1146 \pm 212$ & $1139 \pm 285$ & $1124 \pm 208$ \\
\hline & \multirow{3}{*}{$\begin{array}{l}\text { Switching of } \\
\text { Attention }\end{array}$} & Duration (ms) & $38783 \pm 6742$ & $35235 \pm 6463$ & $38765 \pm 7160$ & $40163 \pm 11671$ \\
\hline & & Connection Time (ms) & $1508 \pm 285$ & $1382 \pm 258$ & $1483 \pm 274$ & $1580 \pm 455$ \\
\hline & & Accuracy & $0 \pm 1$ & $1 \pm 1$ & $1 \pm 2$ & $1 \pm 1$ \\
\hline & Choice RT & Choice RT (ms) & $343 \pm 74$ & $347 \pm 32$ & $329 \pm 35$ & $351 \pm 27$ \\
\hline Response Speed & Motor Tapping & Tapping Speed & $200 \pm 25$ & $199 \pm 23$ & $206 \pm 23$ & $197 \pm 22$ \\
\hline \multirow{3}{*}{$\begin{array}{l}\text { Executive } \\
\text { Function }\end{array}$} & \multirow[t]{3}{*}{ Maze } & Trials Completed & $7 \pm 3$ & $8 \pm 3$ & $8 \pm 2$ & $8 \pm 2$ \\
\hline & & Path Learning Time (ms) & $94322 \pm 38855$ & $94159 \pm 53061$ & $88668 \pm 30494$ & $97255 \pm 38098$ \\
\hline & & Accuracy & $30 \pm 14$ & $28 \pm 11$ & $29 \pm 12$ & $31 \pm 10$ \\
\hline \multirow{2}{*}{$\begin{array}{l}\text { Attention and } \\
\text { Concentration }\end{array}$} & \multirow{2}{*}{$\begin{array}{l}\text { Continuous } \\
\text { Performance Test }\end{array}$} & $\mathrm{RT}$ (ms) & $560 \pm 200$ & $519 \pm 128$ & $467 \pm 69$ & $539 \pm 186$ \\
\hline & & Errors & $3 \pm 6$ & $2 \pm 3$ & $2 \pm 2$ & $4 \pm 7$ \\
\hline \multirow[t]{2}{*}{ Impulsivity } & \multirow[t]{2}{*}{ Go/No-Go } & Speed (ms) & $292 \pm 45$ & $283 \pm 61$ & $288 \pm 44$ & $275 \pm 44$ \\
\hline & & Errors & $1 \pm 2$ & $1 \pm 2$ & $1 \pm 3$ & $2 \pm 3$ \\
\hline
\end{tabular}

RT, reaction time; Visu-I, visual intereference; Verb-I, verbal interference

$* p<0.05$ vs normoxia; $\wedge$ trend, $p<0.10$ vs normoxia 